\title{
Dynamic Tracking Biosensors: Finding Needles in a Haystack Supplementary Material
}

\author{
Deepak Gopalan* and Pradeep R. Nair* \\ Department of Electrical Engineering, Indian Institute of Technology Bombay, Mumbai, India \\ deepak_gopalan@iitb.ac.in, prnair@ee.iitb.ac.in
}

\section{PDF of Dissociation Time}

In the manuscript, we derived several results assuming that the target unbinding time follows as exponential probability distribution. Here, we provide a short proof for the same.

We consider the state transition diagram from Fig. 1b of the manuscript. For a target bound to a receptor (in state $B_{1}$ ) We consider the time it takes for the target molecule to unbind and the receptor to go back to state $U$ as $t_{u b}$. This unbinding time has some probability density (PDF) and an associated cumulative density (CDF). Say the $\mathrm{CDF}$ is given by $F(t)=P\left(t_{u b} \leq t\right)$ and the PDF hence $f(t)=d F / d t$. The CDF at time $t+d t$ is given by $F(t+d t)=P\left(t_{u b} \leq t+d t\right)$. If the unbinding event happens within time $t+d t$, it either happened before $t$ or it happened within the extra $d t$ time. We already know from the state transitions that the probability of the dissociation happening in the $d t$ time is given by $k_{r 1} d t$. Hence, we can say:

$$
\begin{gathered}
F(t+d t)=F(t)+(1-F(t)) k_{r 1} d t \\
\frac{d F(t)}{d t}=\frac{F(t+d t)-F(t)}{d t}=k_{r 1}(1-F(t))
\end{gathered}
$$

We know that $F(0)=0$, hence solving the differential equation gives the $\mathrm{CDF}$ and $\mathrm{PDF}$ as:

$$
\begin{gathered}
F(t)=1-e^{-k_{r 1} t} \\
f(t)=\frac{d F(t)}{d t}=k_{r 1} e^{-k_{r 1} t}
\end{gathered}
$$

The coefficient of $k_{r 1}$ ensures that the total probability of the target dissociation, upon waiting for an infinitely long period of time (integrating the probability distribution from 0 to infinity) converges to 1 as expected. This can also be verified by observing that the CDF converges to 1 as time tends to infinity.

One can also verify that for the PDF derived above, the mean and the standard deviation of the unbinding time, are both $1 / k_{r 1}$

$$
\begin{gathered}
E(t)=\int_{0}^{\infty} t k_{r 1} e^{-k_{r 1} t} d t=\frac{1}{k_{r 1}} \\
\sigma^{2}(t)=\int_{0}^{\infty} t^{2} k_{r 1} e^{-k_{r 1} t} d t-E^{2}(t)=\frac{1}{k_{r 1}^{2}} \\
\mu=\sigma=\frac{1}{k_{r 1}}
\end{gathered}
$$

\section{DEPENDENCE OF DETECTED $N_{1}$ AND $N_{2}$ ON THRESHOLD}

Say in steady-state we have $N_{1}$ and $N_{2}$ receptors bound to target and undesired molecules respectively. Say we are observing the process for a total time of $T$. The fraction of time spent by each receptor in state 1 out of the total time of $\mathrm{T}$ gives us the fraction $N_{1} / N_{0}$.

$$
\begin{gathered}
\frac{N_{1}}{N_{0}}=\frac{\text { time spent by each receptor in state } 1}{T} \\
N_{1}=\frac{\text { time spent by all receptors in state } 1}{T}
\end{gathered}
$$

We already know that the number of unbinding events from state $1, N_{u b}$ is $N_{1} k_{r 1} T$ and that the average unbind time, $\mu_{u b}$ is $1 / k_{r 1}$. We can also say that the time spent by all receptors in state 1 is simply $N_{u b} \mu_{u b}$. These relations show that the equation presented above is indeed valid.

But when we use thresholds to separate state 1 and state 2 , we don't have exact values for $N_{1}$ and $N_{2}$, instead we have $N_{1, \text { det }}, N_{2, \text { det }}$.

$$
N_{1, d e t}=\frac{N_{u b}^{\prime} \mu_{u b}^{\prime}}{T}
$$

$$
\begin{gathered}
N_{u b}^{\prime}=\text { Number of unbinding events with } t_{u b}>t_{t h r} \\
\mu_{u b}^{\prime}=\text { Average unbind time of such events }
\end{gathered}
$$

These events can either be i) Correct detection ii) False positives. We have the number of unbinding events for either case as $N_{1} k_{r 1} T$ and $N_{2} k_{r 2} T$ respectively. We have the probability of each unbinding event taking longer than $t_{t h r}$ for either case as $P_{1}\left(t_{u b}>t_{t h r}\right)$ and $P_{2}\left(t_{u b}>t_{t h r}\right)$. We also have the mean values of unbinding time given the condition as well as $E_{1}$ and $E_{2}$

$$
\text { Say } y_{1}=N_{1} k_{r 1} T P_{1}\left(t_{u b}>t_{t h r}\right) E_{1}\left(t_{u b} \mid t_{u b}>t_{t h r}\right)
$$

$$
\begin{gathered}
\text { Say } y_{2}=N_{2} k_{r 2} T P_{2}\left(t_{u b}>t_{t h r}\right) E_{2}\left(t_{u b} \mid t_{u b}>t_{t h r}\right) \\
N_{1, \text { det }}=\frac{y_{1}+y_{2}}{T}
\end{gathered}
$$

We have

$$
P_{1}\left(t_{u b}>t_{t h r}\right)=\int_{t_{t h r}}^{\infty} k_{r 1} e^{-k_{r 1} t} d t
$$




$$
\begin{gathered}
E_{1}\left(t_{u b} \mid t_{u b}>t_{t h r}\right)=\frac{1}{P_{1}\left(t_{u b}>t_{t h r}\right)} \int_{t_{t h r}}^{\infty} k_{r 1} t e^{-k_{r 1} t} d t \\
E_{1}\left(t_{u b} \mid t_{u b}>t_{t h r}\right) P_{1}\left(t_{u b}>t_{t h r}\right)=\int_{t_{t h r}}^{\infty} k_{r 1} t e^{-k_{r 1} t} d t \\
E_{1}\left(t_{u b} \mid t_{u b}>t_{t h r}\right) P_{1}\left(t_{u b}>t_{t h r}\right)=\frac{1+t_{t h r} k_{r 1}}{k_{r 1}} e^{-k_{r 1} t_{t h r}} \\
E_{2}\left(t_{u b} \mid t_{u b}>t_{t h r}\right) P_{2}\left(t_{u b}>t_{t h r}\right)=\frac{1+t_{t h r} k_{r 2}}{k_{r 2}} e^{-k_{r 2} t_{t h r}} \\
N_{1, d e t}=N_{1} e^{-k_{r 1} t_{t h r}\left(1+k_{r 1} t_{t h r}\right)} \\
+N_{2} e^{-k_{r 2} t_{t h r}}\left(1+k_{r 2} t_{t h r}\right)
\end{gathered}
$$

Similarly,

$$
\begin{gathered}
\text { Say } z_{1}=N_{1} k_{r 1} T P_{1}\left(t_{u b}<t_{t h r}\right) E_{1}\left(t_{u b} \mid t_{u b}<t_{t h r}\right) \\
\text { Say } z_{2}=N_{2} k_{r 2} T P_{2}\left(t_{u b}<t_{t h r}\right) E_{2}\left(t_{u b} \mid t_{u b}<t_{t h r}\right) \\
N_{2, \text { det }}=\frac{z_{1}+z_{2}}{T} \\
E_{1}\left(t_{u b} \mid t_{u b}<t_{t h r}\right) P_{1}\left(t_{u b}<t_{t h r}\right)=\int_{0}^{t_{t h r}} k_{r 1} t e^{-k_{r 1} t_{t h r} t} d t \\
E_{1}\left(t_{u b} \mid t_{u b}<t_{t h r}\right) P_{1}\left(t_{u b}<t_{t h r}\right)=\frac{1}{k_{r 1}}-\frac{1+k_{r 1} t_{t h r}}{k_{r 1}} e^{-k_{r 1} t_{t h r}} \\
N_{2, d e t}=N_{1}\left(1-\left(1+k_{r 1} t_{t h r}\right) e^{\left.-k_{r 1} t_{t h r}\right)}\right. \\
+N_{2}\left(1-\left(1+k_{r 2} t_{t h r}\right) e^{\left.-k_{r 2} t_{t h r}\right)}\right. \\
N_{2, d e t}=N_{1}+N_{2}-N_{1, d e t}
\end{gathered}
$$

Hence we have found expressions for the detected number of receptors bound to the target $\left(N_{1}\right)$ and undesired molecules $\left(N_{2}\right)$ based on the threshold time used for the separation.

\section{Correction SCHEMES WHEN DiSSOCIATION RATES ARE COMPARABLE}

In the main text, we consider the case when the dissociation rates for target and undesired molecules are two or more orders of magnitude apart. The difference in dissociation rates was essential in separating the target molecules form undesired molecules. If the dissociation rates become comparable, we naturally expect the separation procedure to fail. But even with a poor separation, it is still possible to predict the target concentrations accurately. Here, we explore the limits on these parameters so as to still be able to use dynamic tracking.

Consider that, for a chosen threshold, we look at all the unbinding events which last longer than the chosen threshold. Normally, if we assume $k_{r 1}<<k_{r 2}$ then we would simply estimate the $k_{r} 1$ as the mean unbinding time of these events. However, in case the dissociation rates are not well separated, we look for better ways to estimate the $k_{r 1}$ from the unbinding times for a given threshold $t_{t h r}$
Consider the quantity $P$, and $E$ denoting the probability that a particular target-receptor unbinding event is detected and the expectation of the unbinding time for such events. We have:

$$
\begin{gathered}
P=\int_{t_{t h r}}^{T} k_{r 1} e^{-k_{r 1} t} d t \\
E=\frac{1}{P} \int_{t_{t h r}}^{T} t k_{r 1} e^{-k_{r 1} t} d t
\end{gathered}
$$

We consider cases with large enough observation time $(T \rightarrow \infty)$. By solving the above integrals (S-4) and (S-5), We obtain:

$$
E=\frac{1+t_{t h r} k_{r 1}}{k_{r 1}}
$$

Hence we can see that if the quantity $t_{t h r} k_{r 1}$ is not $<<1$, as we would expect ideally, the estimate of $k_{r 1}$ cannot simply be made as the inverse of $E$, which is the mean of those unbinding times which last longer than the threshold. But instead we estimate the target dissociation rate as:

$$
k_{r 1}^{e s t}=\frac{1}{E-t_{t h r}}
$$

Applying this additional correction, we perform a similar analysis as done in the main text, but with parameters: $k_{f 1}=1 e 7, k_{r 1}=1 e-1, \rho_{1}=1 e-8, k_{f 2}=1 e 7, k_{r 2}=1$, $\rho_{2}=1 e-7, N_{0}=1000, T=2000$. The key difference here is that the dissociation constants are only a single order of magnitude apart from each other.

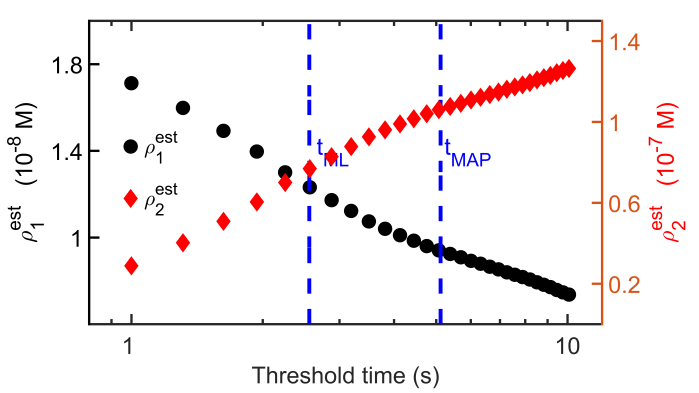

Fig. S-1. Estimated target concentration vs threshold time

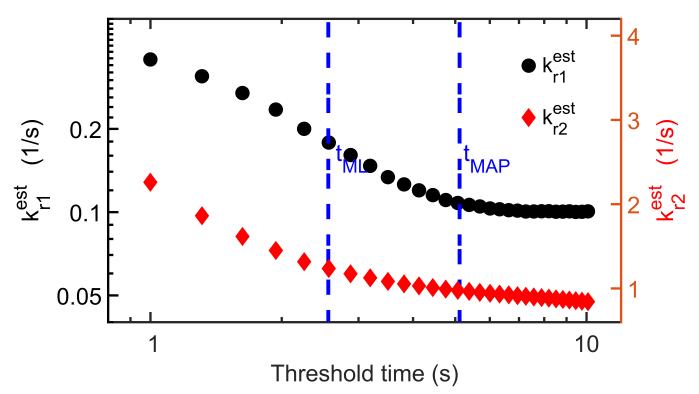

Fig. S-2. Estimated dissociation rates vs threshold time.

Fig. S-2 clearly shows a knee-point region at the ideal MAP threshold. We can see again that the corrected estimate of $k_{r 1}$ matches the ideal value at $t_{M A P}$. However, we do 
TABLE S-I: Drop in Detected Target Concentration due to $k_{r 2}$ and Correction Scheme

\begin{tabular}{|c|c|c|c|c|c|c|c|}
\hline \multirow{2}{*}{$\rho_{2}$} & \multirow{2}{*}{$\mathbf{k}_{\mathrm{r} 2}$} & \multirow{2}{*}{$\begin{array}{c}\text { Naive estimate } \\
\rho_{\mathbf{1}}\left(10^{-10}\right)\end{array}$} & \multicolumn{2}{|c|}{ Detected } & \multicolumn{2}{|c|}{ Corrected } & \multirow{2}{*}{$\begin{array}{c}\text { Corrected estimate } \\
\rho_{\mathbf{1}}\left(10^{-10}\right)\end{array}$} \\
\hline & & & $\mathrm{N}_{1}$ & $\mathbf{N}_{2}$ & $\mathrm{~N}_{1}$ & $\mathbf{N}_{2}$ & \\
\hline \multirow{2}{*}{$10^{-7}$} & 1 & 9.93 & 331.5 & 334.8 & 332.7 & 333.6 & 9.97 \\
\hline & 0.1 & 8.44 & 70.4 & 846.2 & 85.8 & 830.8 & 10.3 \\
\hline \multirow{2}{*}{$10^{-6}$} & 1 & 10 & 83.4 & 833.3 & 83.8 & 832.9 & 10 \\
\hline & 0.1 & 7.45 & 7.3 & 982.9 & 10.0 & 980.2 & 10.2 \\
\hline
\end{tabular}

see an error in the predicted concentration in Fig. S-1 at this ideal $t_{M A P}$ This error can be understood by revisiting equation (S-2) and (S-3). It is clear that when $k_{r 1} t_{M A P}$ is not $<<1$, and $k_{r 2} t_{M A P}$ is not $>>1$, the detected $N_{1}$ and $N_{2}$ are not good approximations of the actual $N_{1}$ and $N_{2}$, even when we choose the ideal threshold of $t_{M A P}$.

A perfect correction requires knowledge of both $k_{r 1}$ and $k_{r 2}$. However, with only knowledge of $k_{r 1}$, we can employ a partial correction scheme. From equations (S-2) and (S-3), we can make the following approximations:

$$
\begin{gathered}
N_{1, \text { det }} \approx N_{1}\left(1+t_{M A P} k_{r 1}\right) e^{-k_{r 1} t_{M A P}} \\
N_{1} \approx N_{1, \text { det }} \frac{e^{k_{r 1} t_{M A P}}}{\left(1+t_{M A P} k_{r 1}\right)} \\
N_{2} \approx N_{1, \text { det }}+N_{2, \text { det }}-N_{1}
\end{gathered}
$$

The results of such a correction is demonstrated by results presented in Table S-1.

Another effect of having the dissociation rates too close, and hence the threshold is too high is that we see a very small fraction of the total unbinding events. The $N_{u b}$ described in the main text as $N_{u b}=\alpha N_{0} k_{r 1} T$ would now be corrected as: $N_{u b}=e^{-k_{r 1} t_{M A P}} \alpha N_{0} k_{r 1} T$, and hence resulting in a new threshold uncertainty given by:

$$
U_{\text {thresh }}=\frac{1}{\sqrt{e^{-k_{r 1} t_{M A P} \alpha N_{0} k_{r 1} T}}} .
$$

One must note here that the exponential nature results in a steep rise in the uncertainty as the dissociation rates get closer, thus making the detection extremely difficult.

\section{Simulations FOR UnCERTAINTY IN THE THRESHOLD TIME}

In the main text, we derived an expression for the uncertainty in threshsold times used in the DT sensors presented in eq. (10). This expression was derived using the central limit theorem and state transition relations. Here, we present the results of statistical simulations which corroborate the expression we derived. First, we study the dependence of uncertainty on the number of receptors $\left(N_{0}\right)$ and the observation time $(T)$.

We consider a scenario with the following reaction parameters: $\rho_{1}=10^{-9} M, \rho_{2}=10^{-7} M, k_{f 1}=10^{7} M^{-1} s^{-1}$, $k_{r 1}=10^{-2} s^{-1}, k_{f 2}=10^{7} M^{-1} s^{-1}, k_{r 2}=1 s^{-1}$. For each choice of $N_{0}$ and $T$ considered in Table S-II, we performed 30 independent simulations of the sensor binding/unbinding events. For each of these iterations, we found the average target unbinding time, $t_{u b}^{a v g}$. Ideally, we would expect each of these 30 average to be $1 / k_{r 1}$. But due to the finite number of receptors and observation time, each iteration results in different observed averages. We obtained the ratio of the standard deviation to the mean of these 30 observed $t_{u b}^{a v g}$. This quantity is presented as the simulated uncertainty.

We also obtain the theoretical uncertainties using eq. (10). We see a close match between the theoretical estimates and simulation results, hence validating the derived expression for uncertainty in threshold time. This dependence of uncertainty on the number of receptors and observation time allows for performance improvement through aggressive scaling.

We performed a similar study for observing the effects of other reaction parameters on uncertainty as well. We set $N_{0}=1000$ and $T=2000$. We also fix the following reaction parameters: $\rho_{1}=10^{-9} M, k_{f 1}=10^{7} M^{-1} s^{-1}, k_{r 1}=10^{-2} s^{-1}$, $k_{f 2}=10^{7} M^{-1} s^{-1}$. We vary both the concentration $\left(\rho_{2}\right)$ and the dissociation rates $\left(k_{r 2}\right)$ of the undesired molecules as shown in Table S-III. The simulated and theoretical uncertainties were obtained using a similar fashion as before.

Note again, that the quantity $\alpha$ in eq. (10) relates both $\rho_{2}$ and $k_{r 2}$ to the uncertainty. As expected, the uncertainty reduces, and detection gets better with a reduction in the undesired molecule concentration and with an increase in the undesired dissociation rate.

TABLE S-II: Threshold uncertainty vs $N_{0}$ and $T$

\begin{tabular}{|c|c|c|c|}
\hline \multirow{2}{*}{$\mathbf{N}_{\mathbf{0}}$} & \multirow{2}{*}{$\mathbf{T}$} & \multicolumn{2}{|c|}{ Uncertainty in Threshold } \\
\cline { 3 - 4 } & & Theoretical & Simulated \\
\hline \multirow{2}{*}{10} & 1000 & $17.43 \%$ & $18.83 \%$ \\
& 5000 & $7.81 \%$ & $6.12 \%$ \\
\hline \multirow{2}{*}{1000} & 1000 & $1.74 \%$ & $1.86 \%$ \\
& 2000 & $1.24 \%$ & $1.54 \%$ \\
\hline
\end{tabular}

TABLE S-III: Threshold uncertainty $v s \rho_{2}$ and $k_{r 2}$

\begin{tabular}{|c|c|c|c|}
\hline \multirow{2}{*}{$\rho_{\mathbf{2}}$} & \multirow{2}{*}{$\mathbf{k}_{\mathbf{r} 2}$} & \multicolumn{2}{|c|}{ Uncertainty in Threshold } \\
\cline { 3 - 4 } & & Theoretical & Simulated \\
\hline \multirow{2}{*}{$10^{-7}$} & 1 & $1.24 \%$ & $1.40 \%$ \\
& 0.1 & $2.45 \%$ & $3.28 \%$ \\
\hline \multirow{2}{*}{$10^{-6}$} & 1 & $2.45 \%$ & $2.66 \%$ \\
& 0.1 & $7.14 \%$ & $6.04 \%$ \\
\hline
\end{tabular}

